\title{
An Improved Lower Bound for Crossing Numbers
}

\author{
Hristo Djidjev ${ }^{1}$ and Imrich Vrto ${ }^{2, \star}$ \\ ${ }^{1}$ Department of Computer Science, Warwick University \\ Coventry CV4 7AL, United Kingdom \\ hristo@dcs.warwick.ac.uk \\ 2 Department of Informatics, Institute of Mathematics \\ Dúbravská 9, 84235 Bratislava, Slovak Republic \\ vrto@savba.sk
}

\begin{abstract}
The crossing number of a graph $G=(V, E)$, denoted by $\operatorname{cr}(G)$, is the smallest number of edge crossings in any drawing of $G$ in the plane. Leighton [14] proved that for any $n$-vertex graph $G$ of bounded degree, its crossing number satisfies $\operatorname{cr}(G)+n=\Omega\left(\mathrm{bw}^{2}(G)\right)$, where bw $(G)$ is the bisection width of $G$. The lower bound method was extended for graphs of arbitrary vertex degrees to $\operatorname{cr}(G)+\frac{1}{16} \sum_{v \in G} d_{v}^{2}=$ $\Omega\left(\mathrm{bw}^{2}(G)\right)$ in [1519], where $d_{v}$ is the degree of any vertex $v$. We improve this bound by showing that the bisection width can be replaced by a larger parameter - the cutwidth of the graph. Our result also yields an upper bound for the path-width of $G$ in term of its crossing number.
\end{abstract}

\section{Introduction}

The crossing number of a graph $G=(V, E)$, denoted by $\operatorname{cr}(G)$, is the smallest number of edge crossings in any drawing of $G$ in the plane. It represents a fundamental measure of non-planarity of graphs and has been studied for more than 40 years. The problem is attractive from practical point of view too. It is known that the aesthetics and readability of graph-like structures (information diagrams, class hierarchies, flowcharts...) heavily depends on the number of crossings 4 16, when the structures are visualized on a 2-dimensional medium. Another natural appearance of the problem is in the design of printed circuit boards and VLSI circuits [14. The area of a VLSI circuit is strongly related to the crossing number of the underlying graph. The problem is NP-hard [7] and the best theoretical exact and approximation algorithms are in [510]. A survey on heuristics is in [3]. Concerning crossing numbers of standard graphs, there are only a few infinite classes of graphs for which exact or tight bounds are known 13. The main problem is the lack of efficient lower bound methods for estimating the crossing numbers of explicitely given graphs. The survey on known methods is in [17. One of the powerful methods is based on the bisection

\footnotetext{
* This research was supported by the VEGA grant No. 02/7007/20 and the DFG grant No. Hr14/5-1.
}

P. Mutzel, M. Jünger, and S. Leipert (Eds.): GD 2001, LNCS 2265, pp. 96-101, 2002.

(C) Springer-Verlag Berlin Heidelberg 2002 
width concept. The bisection width of a graph $G$ is the minimum number of edges whose removal divides $G$ into two parts having at most $2|V| / 3$ vertices each. Leighton [14] proved that in any $n$-vertex graph $G$ of bounded degree, the crossing number satisfies $\operatorname{cr}(G)+n=\Omega\left(\mathrm{bw}^{2}(G)\right)$. The lower bound was extended to

$$
\operatorname{cr}(G)+\frac{1}{16} \sum_{v \in V} d_{v}^{2} \geq \frac{1}{40} \mathrm{bw}^{2}(G)
$$

in 1519 , where $d_{v}$ is the degree of any vertex $v$. We improve this bound by showing that the bisection width can be replaced by a larger parameter - the cutwidth of the graph, defined as follows. Consider an injection of the vertices of $G$ into points of a horizontal line. Draw the edges above the line using semicircles. Find a vertical line between a pair of consecutive points, which cuts the maximal number of edges. Minimize the maximum over all injections. The minmax value is called the cutwidth of $G$, denoted by $\mathrm{cw}(G)$. Note that the cutwidth is a standard graph invariant appearing e.g. in the linear VLSI layouts [20, and is related to such a classical topic like the discrete isoperimetric problem [1]. We prove

$$
\operatorname{cr}(G)+\frac{1}{16} \sum_{v \in G} d_{v}^{2} \geq \frac{1}{1176} \mathrm{cw}^{2}(G) .
$$

Regardless the constant factors, the improvement is evident as $\mathrm{cw}(G) \geq \mathrm{bw}(G)$ and there are connected graphs with bw $(G)=1$ but with arbitrarily large cutwidth. If $\operatorname{cw}(G) \approx \operatorname{bw}(G)$ then the bisection lower bound is better up to a constant factor because of the big constant in our estimation. An improvement on it remains an open problem. Anyway, the aim of this note is to show that from the asymptotical point of view, the graph invariant that essentially influences the crossing number is not the bisection width but the cutwidth. The new crossing number lower bound is tight up to a constant factor. E.g., the cutwidth of the $n$-cube $Q_{n}$ is $\left\lceil 2^{n+1} / 3\right\rceil$, see [2], which implies

$$
\operatorname{cr}\left(Q_{n}\right)=\Omega\left(4^{n}\right),
$$

while it is known that $\operatorname{cr}\left(Q_{n}\right) \geq 4^{n} / 20-O\left(n^{2} 2^{n}\right)$, 18] and the best upper bound for the crossing number of the hypercube graph is $(163 / 1024) 4^{n}, 6$.

Moreover, the additive term $\sum_{v \in G} d_{v}^{2}$ can not be removed, since the crossing number of any planar graph is 0 and there exists a planar graph $P$ (e.g. the star) such that $\mathrm{cw}^{2}(P)=\Omega\left(\sum_{v \in P} d_{v}^{2}\right)$.

As a byproduct of our result we obtain the following contribution to the topological graph theory. The path-decomposition of a graph $G$ is a sequence $D=X_{1}, X_{2}, \ldots, X_{r}$ of vertex subsets of $G$, such that every edge of $G$ has both ends in some set $X_{i}$ and if a vertex of $G$ occurs in some sets $X_{i}$ and $X_{j}$ with $i<j$, then the same vertex occurs in all sets $X_{k}$ with $i<k<j$. The width of $D$ is the maximum number in any $X_{i}$ minus 1 . The path-width of $G, \operatorname{pw}(G)$, is the minimum width over all path-decompositions of $G$.

A graph $G=(V, E)$ is $k$-crossing critical if $\operatorname{cr}(G)=k$ and $\operatorname{cr}(G-e)<\operatorname{cr}(G)$, for all edges $e \in E$. Hliněný [11] proved that $\mathrm{pw}(\mathrm{G}) \leq 2^{f(k)}$, where $f(k)=$ 
$O\left(k^{3} \log k\right)$. This answers an open question of Geelen et al. 9] whether crossing critical graphs with bounded crossing numbers have bounded path-widths.

From the practical point of view, as $\mathrm{pw}(\mathrm{G}) \leq n-1$, the above upper bound is useful for $k \leq \sqrt[3]{\log n}$ only, while the maximal order of crossing numbers is $n^{4}$. Our result implies that if $\operatorname{cr}(G)=k$, then $\operatorname{pw}(\mathrm{G})=O\left(\sqrt{k+\sum_{v \in V} d_{v}^{2}}\right)$, without the crossing-criticality assumption.

\section{A New Lower Bound}

We will make use of the following theorem [8].

Theorem 1. Let $G=(V, E)$ be a planar graph with non-negative weights on its vertices that sum up to one and every weight is at most $2 / 3$. Let $d_{v}$ is the degree of any vertex $v$. Then there exists at most $(\sqrt{3}+\sqrt{2}) \sqrt{\sum_{v \in V} d_{v}^{2}} / 2$ edges whose removal divides $G$ into disjoint subgraphs $G_{1}=\left(V_{1}, E_{1}\right)$ and $G_{2}=\left(V_{2}, E_{2}\right)$ such that the weight of each is at most $2 / 3$.

Theorem 1 implies an upper bound for the cutwidth of planar graphs which deserves an independent interest.

Theorem 2. For any planar graph $G=(V, E)$

$$
\operatorname{cw}(G) \leq \frac{6 \sqrt{2}+5 \sqrt{3}}{2} \sqrt{\sum_{v \in V} d_{v}^{2}},
$$

where $d_{v}$ is the degree of any vertex $v$.

Proof. Apply Theorem 11 to $G$. Assign weights to vertices:

$$
\operatorname{weight}(u)=\frac{d_{u}^{2}}{\sum_{v \in V} d_{v}^{2}} .
$$

1. Assume weight $(u) \leq 2 / 3$ for all $u$. By deleting $(\sqrt{3}+\sqrt{2}) \sqrt{\sum_{v \in V} d_{v}^{2}} / 2$ edges we get graphs $G_{1}=\left(V_{1}, E_{1}\right)$ and $G_{2}=\left(V_{2}, E_{2}\right)$ such that for $i=1,2$ weight $\left(V_{i}\right) \leq 2 / 3$, which implies

$$
\sum_{v \in V_{i}} d_{v}^{2} \leq \frac{2}{3} \sum_{v \in V} d_{v}^{2}
$$

2. Assume there exists a vertex $u$ such that weight $(u)>2 / 3$. By deleting edges adjacent to $u$ we get disjoint subgraphs $G_{1}=\left(V_{1}, E_{1}\right)$ and $G_{2}=\left(V_{2}, E_{2}\right)$, where $G_{2}$ is a one vertex graph. We have weight $\left(V_{1}\right)=1-\operatorname{weight}(u)<2 / 3$ and

$$
\sum_{v \in V_{1}} d_{v}^{2}<\frac{2}{3} \sum_{v \in V} d_{v}^{2}
$$

The number of edges between $G_{1}$ and $G_{2}$ is

$$
d_{u} \leq \frac{\sqrt{3}+\sqrt{2}}{2} \sqrt{\sum_{v \in V} d_{v}^{2}}
$$


Placing the graphs $G_{1}$ and $G_{2}$ consecutively on the line and adding the deleted edges we obtain the estimation

$$
\mathrm{cw}(G) \leq \max \left\{\operatorname{cw}\left(G_{1}\right), \operatorname{cw}\left(G_{2}\right)\right\}+\frac{(\sqrt{3}+\sqrt{2})}{2} \sqrt{\sum_{v \in V} d_{v}^{2}} .
$$

Solving the recurrence we find

$$
\operatorname{cw}(G) \leq \frac{\sqrt{3}+\sqrt{2}}{2} \sum_{i=0}^{\infty}\left(\frac{2}{3}\right)^{i / 2} \sqrt{\sum_{v \in V} d_{v}^{2}}=\frac{6 \sqrt{2}+5 \sqrt{3}}{2} \sqrt{\sum_{v \in V} d_{v}^{2}} .
$$

Our main result is

Theorem 3. Let $G=(V, E)$ be a graph. Let $d_{v}$ denote the degree of any vertex $v$. Then the crossing number of $G$ satisfies

$$
\operatorname{cr}(G)+\frac{1}{16} \sum_{v \in V} d_{v}^{2} \geq \frac{1}{1176} \mathrm{cw}^{2}(G) .
$$

Proof. Consider a drawing of $G$ with $\operatorname{cr}(G)$ crossings. Introducing a new vertex at each crossing results in a plane graph $H$ with $\operatorname{cr}(G)+n$ vertices. By Theorem 2 we have

$$
\mathrm{cw}(H) \leq \frac{6 \sqrt{2}+5 \sqrt{3}}{2} \sqrt{\sum_{v \in H} d_{v}^{2}}=\frac{6 \sqrt{2}+5 \sqrt{3}}{2} \sqrt{\sum_{v \in G} d_{v}^{2}+16 \mathrm{cr}(G)} .
$$

Finally, note that $\mathrm{cw}(G) \leq \mathrm{cw}(H)$, which proves the claim.

This result immediately gives an upper bound for the path-width of $G$ in term of its crossing number as the result of Kinnersley [12] implies that $\operatorname{pw}(G) \leq$ $\mathrm{cw}(G)$.

Corollary 1. Let $G=(V, E)$ be a graph. Then

$$
\operatorname{pw}(G)<9 \sqrt{16 \operatorname{cr}(G)+\sum_{v \in V} d_{v}^{2}} .
$$

\section{Final Remarks}

We proved a new lower bound formula for estimating the crossing numbers of graphs. The former method was based on the bisection width of graphs. Our method replaces the bisection width by a stronger parameter - the cutwidth. While the bisection width of a connected graph can be just one edge, which implies a trivial lower bound only, the cutwidth based method gives nontrivial lower bounds in most cases. A drawback of the method is the big constant factor in the formula. Currently we are able to reduce it to about 500 . 
A natural question arises how to find or estimate the cutwidth of a graph. The most frequent approach so far was its estimation from below by the bisection width. This of course degrades the cutwidth method to the bisection method. Provided that $c w(H)$ in known or estimated from below, for some graph $H$, we can use a well-known relation $c w(G) \geq c w(H) / c g(H, G)$, where $c g(H, G)$ is the congestion of $G$ in $H$. Another possibility is to use the strong relation of the cutwidth problem to the so called discrete edge isoperimetric problem 1 . Informally, the problem is to find, for a given $k$, a $k$-vertex subset of a graph with the smallest "edge boundary". A good solution to the isoperimetric problem provides a good lower bound for the cutwidth.

\section{References}

1. Bezrukov, S.L.,: Edge Isoperimetric Problems on Graphs. In: Lovász, L., Gyarfás, A.,Katona, G.O.H., Recski, A., Székely, L. (eds.): Graph Theory and Combinatorial Biology. Bolyai Soc. Mathematical Studies 7. Akadémia Kiadó, Budapest (1999) 157-197

2. Bezrukov, S., Chavez, J.D., Harper, L.H., Röttger, M., Schroeder, U.-P.: The Congestion of n-Cube Layout on a Rectangular Grid. Discrete Mathematics 213 (2000) 13-19

3. Cimikowski, R.: Algorithms for the Fixed Linear Crossing Number Problem. Submitted to Discrete Applied Mathematics

4. Di Battista, G., Eades, P., Tamassia, R., Tollis, I.G.: Graph Drawing: Algorithms for Visualization of Graphs. Prentice Hall (1999)

5. Even, G., Guha, S., Schieber, B.: Improved Approximations of Crossings in Graph Drawing and VLSI Layout Area. In: 32th Annual Symposium on Theory of Computing. ACM Press (2000) 296-305

6. Faria, L., Herrera de Figuerado, C.M.: On Eggleton and Guy conjectured upper bounds for the crossing number of the $n$-cube. Mathematica Slovaca 50 (2000) 271-287

7. Garey, M. R., and Johnson, D. S.: Crossing Number is NP-complete. SIAM J. Algebraic and Discrete Methods 4 (1983) 312-316

8. Gazit, H., Miller, G.L.: Planar Separators and the Euclidean Norm. In: SIGAL Intl. Symposium on Algorithms. Lecture Notes in Computer Science, Vol. 450. Springer Verlag, Berlin (1990) 338-347

9. Geelen, J. F., Richter, R.B., Salazar, G.: Embedding Graphs on Surfaces. Submitted to J. Combinatorial Theory-B

10. Grohe, M.: Computing Crossing Numbers in Quadratic Time. In: 33rd Annual ACM Symposium on Theory of Computing. ACM Press (2001)

11. Hliněný, P.: Crossing-Critical Graphs and Path-Width. In: 9th Intl. Symposium on Graph Drawing. Lecture Notes in Computer Science, Springer Verlag, Berlin (2001)

12. Kinnersley, N.: The Vertex Separation Number of a Graph Equals its Path-Width. Information Processing Letters 142 (1992) 345-350

13. Liebers, A.: Methods for Planarizing Graphs - a Survey and Annotated Bibliography. J. of Graph Algorithms and Applications 5 (2001) 1-74

14. Leighton, F. T.: Complexity Issues in VLSI, M.I.T. Press, Cambridge (1983)

15. Pach, J., Shahrokhi, F., Szegedy, M.: Applications of Crossing Number. Algorithmica 16 (1996) 111-117 
16. Purchase, H.: Which Aestethic has the Greatest Effect on Human Understanding? In: 5th Intl. Symposium on Draph Drawing. Lecture Notes in Computer Science, Vol. 1353. Springer Verlag, Berlin, (1997) 248-261

17. Shahrokhi, F., Sýkora, O., Székely, L.A., Vrto, I.: Crossing Numbers: Bounds and Applications. In: Bárány, I., Boroczky, K. (eds.): Intuitive Geometry. Bolyai Society Mathematical Studies 6. Akadémia Kiadó, Budapest (1997) 179-206

18. Sýkora, O., Vrto, I.: On the Crossing Number of the Hypercube and the Cube Connected Cycles. BIT 33 (1993) 232-237

19. Sýkora, O., Vrto, I.: On VLSI Layouts of the Star Graph and Related Networks. Integration, The VLSI Journal 17 (1994) 83-93

20. Wei-Liang Lin, Amir H. Farrahi, A. H., Sarrafzadeh, M.: On the Power of Logic Resynthesis. SIAM J. Computing 29 (2000) 1257-1289 